\title{
The effect of dexamethasone on lentiviral vector infection is associated with importin $\alpha$
}

\author{
SHENGCHANG DENG ${ }^{1}$, YING ZHOU ${ }^{1}$, DONG OUYANG ${ }^{2}$, JUNPING XIONG ${ }^{1}$, LEI ZHANG ${ }^{1}$, \\ CHANGCHUN TU ${ }^{1}$, KEPING ZHANG ${ }^{1}$, ZENGLIANG SONG ${ }^{3}$ and FANGLIN ZHANG ${ }^{1}$ \\ ${ }^{1}$ School of Medicine, Nanchang University, Nanchang, Jiangxi 330006; ${ }^{2}$ Jiangxi Police College, Nanchang, Jiangxi 330103; \\ ${ }^{3}$ Department of Neurosurgery, The Third People's Hospital of Nanchang, Nanchang, Jiangxi 330009, P.R. China
}

Received September 27, 2013; Accepted October 30, 2013

DOI: $10.3892 /$ br.2013.194

\begin{abstract}
Importin $\alpha(\operatorname{Im} \alpha)$ plays an important role during the shuttling of the HIV-1 preintegration complex (PIC) from the cytoplasm to the nucleus. Im $\alpha$ may bind to the glucocorticoid receptor (GR), which is localized to nucleus following hormone binding. However, it remains unclear whether the binding of dexamethasone (Dex) to GR affects the Im $\alpha$ redistribution and, thus, alters PIC import. In our study, 293T cells were transfected with the lentiviral vector (LV) carrying the luciferase (Luci) gene following Dex or RU486 pretreatment. The Luci activity (LucA) in the Dex or RU486 group was significantly higher compared to that in the control group $(\mathrm{P} \leq 0.01)$. The effects of Dex and RU486 were inhibited by the Im $\alpha$ inhibitor Bimax1 $(\mathrm{P} \leq 0.01)$, although the inhibitory effect of Bimax1 was alleviated by increasing the Dex dose. Furthermore, it was observed that the LucA in the 30-min Dex treatment group was lower compared to that in the 30-min Dex pretreatment group $(\mathrm{P} \leq 0.01)$. These results suggested that Dex may improve PIC import via increasing the cytoplasmic Ima levels. Kunming mice were transfected in vivo with the LV, either $30 \mathrm{~min}$ or $15 \mathrm{~h}$ following an intraperitoneal injection of Dex. The LucA in the liver of the 30-min group mice was significantly lower compared to that of the 15-h group mice $(\mathrm{P} \leq 0.01)$, suggesting that the effect of Dex on LV infection depends mainly on the suppression of immune and inflammatory responses in vivo. Taken together, our data indicated that the effect of Dex on LV infection may be associated with Im $\alpha$, constituting a novel signaling pathway mediating the effects of Dex on HIV-1 infection.
\end{abstract}

Correspondence to: Dr Fanglin Zhang, School of Medicine, Nanchang University, Nanchang, Jiangxi 330006, P.R. China E-mail: zhangf105@gmail.com

Key words: lentiviral vector, importin $\alpha$, glucocorticoid receptor, dexamethsone, mouse

\section{Introduction}

The capsid of the lentiviral vector (LV) is disassembled shortly after LV is endocytosed into the cytoplasm and the retrotranscription process is initiated, producing a double-strand viral DNA. This double-strand viral DNA, together with matrix protein (MA), integrase (IN), HIV-1 viral protein R (Vpr) and cellular factors, form the preintegration complex (PIC). Subsequently, PIC binds to importin $\alpha(\operatorname{Im} \alpha)$ through the interaction of Im $\alpha$ with the nuclear location signal (NLS) located in MA and IN (1-4). Furthermore, Vpr was shown to increase the Im $\alpha$ affinity for NLS (5).

The Im $\alpha$ carrying PIC is then combined with importin $\beta$, forming the Im $\alpha / \beta$ heterodimer. The Im $\alpha / \beta$ interacts with a nucleoporin of phenylalanine-glycine repeats, leading to PIC import (6). Subsequently, PIC is disassembled in the nucleus through the binding of RanGTP to importin $\beta$, resulting in the separation of PIC from Im $\alpha$. The double-strand viral DNA is then integrated into the target cell genome to achieve LV infection.

The glucocorticoid receptor (GR) is also combined with Im $\alpha$ and transported to the nucleus, even in the absence of glucocorticoid (3). GR has two NLSs and one nuclear export signal. The first NLS (NLS-1), which is located in the DNA-binding domain and is similar to the SV40 NLS, binds Im $\alpha$. The second NLS (NLS-2), which is located in the ligand-binding domain, binds glucocorticoid. GR is localized to the cytoplasm in the absence of hormone and localizes to the nucleus following hormone binding (3). Since GR is able to bind Im $\alpha$, GR retention in the nucleus may theoretically result in Im $\alpha$ redistribution, affecting PIC import.

In the cytoplasm, GR transports transcription factors to the nucleus and alters their activity (e.g., nuclear factor $N F-\kappa B$ ), triggering gene transcription modulation (7). In the nucleus, GR may directly bind to a specific DNA sequence, referred to as glucocorticoid response element (GRE), which is a short sequence of DNA within the promoter of a gene that is able to bind the GR complex and regulate transcription. Depending on the cell line, glucocorticoids may differentially affect HIV-1 expression (8).

Despite the well-known effect of dexamethasone (Dex) on HIV-1 gene transcription, it has not thus far been determined whether the binding of Dex to GR affects the Im $\alpha$ redistribu- 
tion. This study was designed to confirm the Im $\alpha$ redistribution induced by Dex through detecting the luciferase (Luci) activity (LucA) in cells transfected with the Luci reporter LV. The Ima redistribution affects $\mathrm{LV}$ infection, as well as other nucleophilic imports.

\section{Materials and methods}

Luci expression vector construct. The Luci gene was obtained from the pGl3 plasmid (Promega, Madison, WI, USA) by a polymerase chain reaction and cloned into $\mathrm{pcDNA}^{\mathrm{TM}}$ 6.2-GW/miR (Invitrogen, Carlsbad, CA, USA) to form pcDNA ${ }^{\mathrm{TM}} 6.2-\mathrm{GW} /$ Luci. pcDNA6.2-GW/Luci was then recombined with $\mathrm{pDONR}^{\mathrm{TM}} 221$ (Invitrogen) to generate a $\mathrm{pDONR}{ }^{\mathrm{TM}}$ Luci entry clone (BP recombination). The pDONR Luci entry clone was again recombined with pLenti6/V5-DEST ${ }^{\mathrm{TM}}$ (Invitrogen) to construct pLenti6/Luci (LP recombination). Subsequently, the products of the LP recombination were treated with proteinase $K$ and then transformed into One Shot ${ }^{\circledR}$ Stbl3 ${ }^{\mathrm{TM}}$ Chemically Competent E. coli to obtain pLenti6/Luci (Fig. 1). pLenti6/Luci was identified with nucleic acid electrophoresis and LucA assay.

\section{LV preparation}

Preparation of the DNA complex. A total of $9 \mu \mathrm{g}$ of ViraPower ${ }^{\mathrm{TM}}$ Packaging Mix (Invitrogen) and $3 \mu \mathrm{g}$ of pLenti6/Luci were added to $1.5 \mathrm{ml}$ Dulbecco's modified Eagle's medium (DMEM; Sigma, St. Louis, MO, USA) without serum and mixed gently.

Preparation of the GenEscort U complex. A total of $36 \mu 1$ GenEscort II (Wisegen Biotechnology Corp., Nanjing, China) were diluted in $1.5 \mathrm{ml}$ DMEM without serum, mixed gently and incubated for $5 \mathrm{~min}$ at room temperature.

Preparation of the transfection complex. The DNA complex was added to the GenEscort III complex, mixed gently and incubated for $20 \mathrm{~min}$ at room temperature. At the same time, the $293 \mathrm{~T}$ cells were resuspended in DMEM at a density of $1.2 \times 10^{6}$ cells $/ \mathrm{ml}$. Subsequently, DNA-GenEscort U was added to a $10-\mathrm{cm}$ tissue culture plate containing $5 \mathrm{ml}$ DMEM supplemented with $10 \%$ fetal bovine serum, $2 \mathrm{mM}$ L-glutamine, $0.1 \mathrm{mM}$ minimum essential medium (MEM) non-essential amino acids and $1 \mathrm{mM}$ MEM sodium pyruvate. The $293 \mathrm{~T}$ cell suspension $(5 \mathrm{ml})$ was added to the plate and mixed by gentle rocking. Finally, the cells were incubated overnight at $37^{\circ} \mathrm{C}$ in a $\mathrm{CO}_{2}$ incubator. The following day, the medium containing DNA-GenEscort III was removed and replaced with complete culture medium. The supernatants were harvested 48-72 $\mathrm{h}$ after transfection, centrifuged at $1,000 \mathrm{x}$ g for $5 \mathrm{~min}$ at $4^{\circ} \mathrm{C}$ and stored at $-80^{\circ} \mathrm{C}$. The $\mathrm{LV}$ was titered immediately prior to use. All the operations applied to Biosafety Level 2.

Cell culture and pretreatment. The $293 \mathrm{~T}$ cells $\left(1 \times 10^{3} /\right.$ well) were maintained in 96-well plates containing $200 \mu \mathrm{l}$ DMEM (as described above) and each well was repeated 3 times. RU486 (1×10-6 M; Sigma) was used for stimulating GR shuttling to the nucleus (9). Bimax1 $\left(2.5 \times 10^{-8} \mathrm{M}\right.$; Shanghai Science Peptide Biological Technology Co., Ltd, Shanghai, China) was used for inhibiting Im $\alpha(10,11)$. Pretreatment with Dex (Sigma) was classified into 0-, 5-, 15-, 30-, 60- and 120-min groups (12); the grouping for Dex treatment was the same as that for Dex pretreatment. The 30-min group of Dex pretreat-
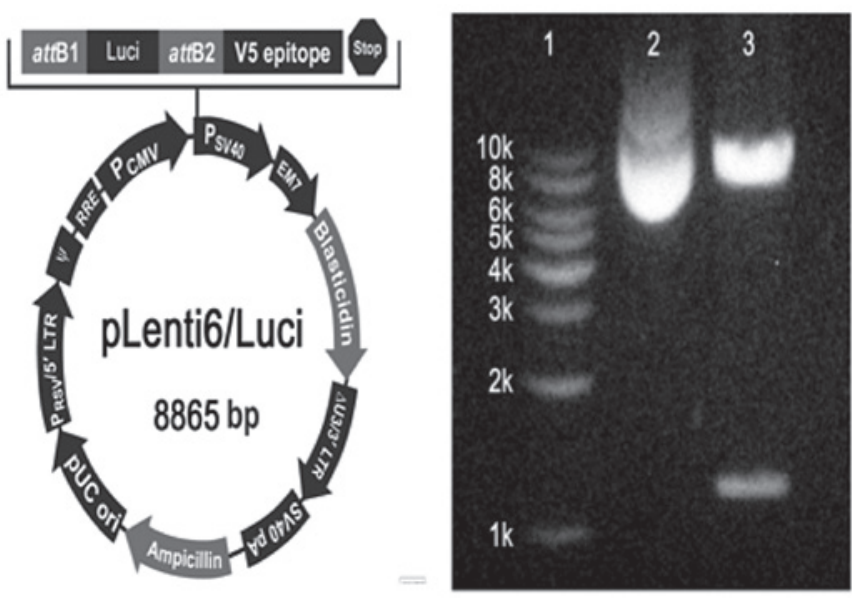

Figure 1.pLenti6/Luci map (left panel) and electrophoretogram (right panel). Lane 2, pLenti6/Luci; and lane 3, pLenti6/Luci digested by $B \ln I$.

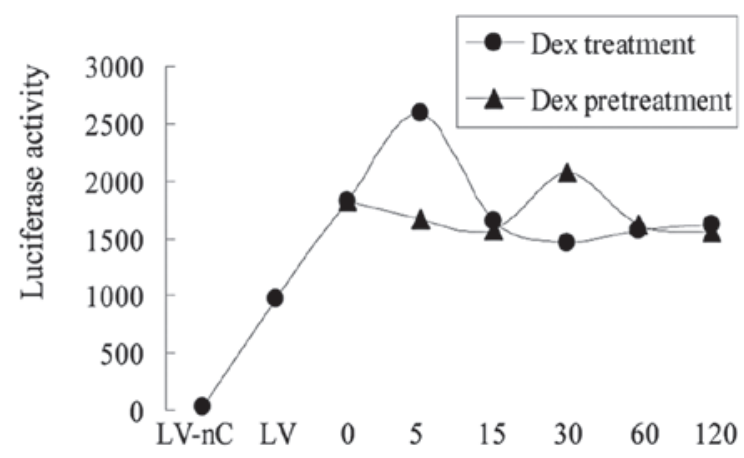

Figure 2. The effect of dexamethasone (Dex) on luciferase activity. The difference between the lentiviral vector (LV) and the 0 -min group was $\mathrm{P} \leq 0.01$. Upon Dex pretreatment, the difference between the 30- and the 15- or 60-min groups was $\mathrm{P} \leq 0.01$. Upon Dex treatment, the difference between the 5- and the $0-, 15-, 30-, 60$ - or $120-\mathrm{min}$ groups was $\mathrm{P} \leq 0.01$. The difference between the 30 -min Dex treatment and pretreatment groups was $\mathrm{P} \leq 0.01$. LV-nC was used as a negative control.

ment was again classified into two dose groups of $1 \times 10^{-7}$ and $1 \times 10^{-6} \mathrm{M}$ (Dex-1 and Dex-2, respectively) (13). All the cells were used for LucA assay $72 \mathrm{~h}$ after pretreatment or treatment.

Animals. An amount of $0.5 \mathrm{mg} / 10 \mathrm{~g}$ Dex and LV (titer $=10^{6}$ ) was administered by intraperitoneal injection (14). A total of 42 Kunming mice of clean grade (half of the animals were female, although the gender of the animal was not considered a significant factor) were randomly assigned into 7 groups as follows: LV-nC (LucA-negative control); LV-1C [20 $\mu \mathrm{l} \mathrm{LV/mouse,} \mathrm{normal} \mathrm{saline} \mathrm{(NS)} \mathrm{pretreatment} \mathrm{control];}$ LV-2C (100 $\mu \mathrm{l} \mathrm{LV} /$ mouse, NS pretreatment control); LV-1/P-1 (20 $\mu \mathrm{l} \mathrm{LV} /$ mouse, 30-min Dex pretreatment); LV-2/P-1 (100 $\mu \mathrm{l} \mathrm{LV/mouse,} \mathrm{30-min} \mathrm{Dex} \mathrm{pretreatment);} \mathrm{LV-1/P-2} \mathrm{(20} \mu \mathrm{l}$ LV/mouse, 15-h Dex pretreatment); LV-2/P-2 (100 $\mu$ LV/mouse, 15-h Dex pretreatment). Seven days after the injection of LV, the mice were anaesthetized with $0.4 \%$ pentobarbital sodium and sacrificed and their livers were immediately excised. The mice were maintained and handled in accordance with the National and International Guiding Principles for Biomedical Research Involving Animals, as promulgated by the Society for the Study of Reproduction. 


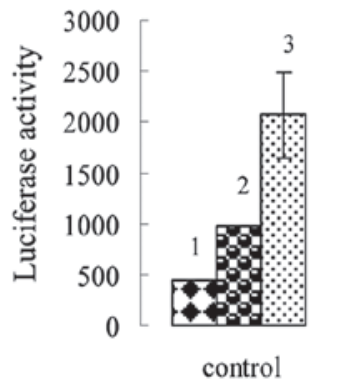

Figure 3. The effect of Bimax1 on importin $\alpha(\operatorname{Im} \alpha)$ was alleviated by Dex. Control 1: the cells were pretreated with Bimax1 30 min prior to LV transfection; control 2: the cells were transfected with LV alone; control 3: the cells were pretreated with Dex 30 min prior to LV transfection (difference between control 1 and control 2 and 3,P $\leq 0.01$ ); Dex-1 and Dex-2 group: the cells were pretreated by Bmax 1, followed by the addition of $1 \times 10^{-7}$ and $1 \times 10^{-6} \mathrm{M}$ Dex respectively, $30 \mathrm{~min}$ prior to LV transfection (difference between Dex-1 and Dex-2 groups, $\mathrm{P} \leq 0.01$ ).

LucA assay. The 293T cells and $0.2 \mathrm{~g}$ of mouse liver were lysed in cell culture lysis reagent (Promega) at $-80^{\circ} \mathrm{C}$ for $30 \mathrm{~min}$ and then immersed in $37^{\circ} \mathrm{C}$ water bath for $5 \mathrm{~min}$. Subsequently, the lysates were collected and centrifuged at 13,800 $\mathrm{x}$ g for $2 \mathrm{~min}$ at $4^{\circ} \mathrm{C}$ to remove cell debris. The LucA in $100 \mu 1$ of lysate was assessed by the addition of $100 \mu \mathrm{l}$ Luci assay reagent (Promega). After $30 \mathrm{~min}$ of pre-incubation, the produced light was measured for $10 \mathrm{sec}$ with the GloMax ${ }^{\circledR}$-Multi Jr Single Tube Multimode Reader (Promega).

Statistical analysis. Data were analyzed for significant differences by the independent samples t-test. A P $<0.05$ was considered to indicate a statistically significant difference. The tests were performed with SPSS software, version 10.0 for Windows (SPSS, Inc., Chicago, IL, USA). All the values are presented as means \pm standard deviation.

\section{Results}

Effect of Dex on LucA (Fig. 2). Considering that Dex results in GR retention in the nucleus, it may be hypothesized that cytoplasmic Im $\alpha$ is decreased due to the ability of GR to bind Im $\alpha$, which suggests that Dex pretreatment may inhibit PIC import. To determine whether Dex affects PIC import, the 293T cells were pretreated with Dex. As a result, the LucA in the Dex pretreatment group was significantly higher compared to that in the LV alone group $(\mathrm{P} \leq 0.01)$. Furthermore, the LucA in the 30-min Dex pretreatment group was the highest, with a significant difference between the 30-min Dex pretreatment group and the 15 - or the 60 -min groups $(\mathrm{P} \leq 0.01)$. However, these results were not sufficient to determine whether cytoplasmic Im $\alpha$ is decreased even if Dex improves LV transcription. To verify that Dex improves LV transcription, the 293T cells were treated with Dex following transfection. The LucA in the Dex treatment groups was significantly higher compared to that in the LV alone group ( $\mathrm{P} \leq 0.01)$, with the LucA in the 5-min Dex treatment group being the highest. Of note, the LucA in the 5-min Dex treatment group was higher compared to that in the 5-min Dex pretreatment group; however, the LucA in 30-min Dex treatment group was lower compared to that in the 30-min pretreatment group $(\mathrm{P} \leq 0.01)$, which suggests that pretreatment for 30 min with Dex improves PIC import as well

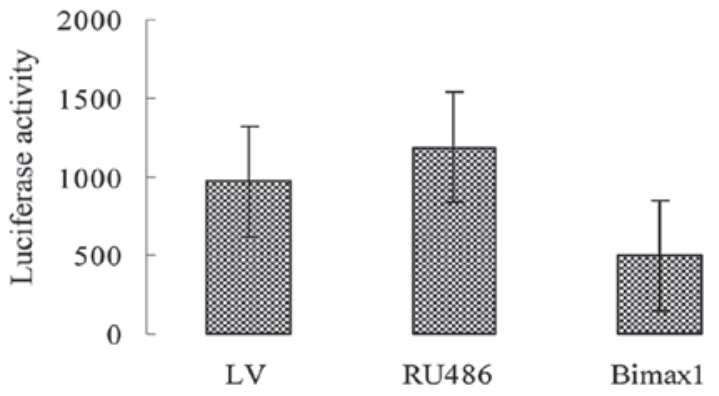

Figure 4. Effect of RU486 on importin $\alpha(\operatorname{Im} \alpha)$. RU486 group: the cells were pretreated with RU486 30 min prior to transfection; Bimax1 group: the cells were pretreated with RU486, followed by the addition of Bmax1 30 min prior to LV transfection (difference between RU486 and Bimax1 groups, $\mathrm{P} \leq 0.01$ ).

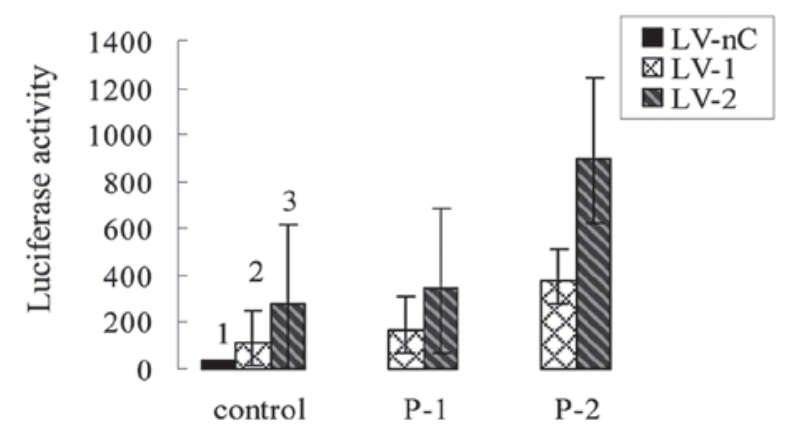

Figure 5. Effect of dexamethasone (Dex) on LucA in vivo. LV-1C: $20 \mu 1$ $\left(\right.$ titer $\left.=10^{6}\right) \mathrm{LV} /$ mouse, normal saline (NS) pretreatment; LV-2C: $100 \mu 1$ (titer $=10^{6}$ ) LV/mouse, NS pretreatment; P-1: Dex pretreatment for $30 \mathrm{~min}$; P-2: Dex pretreatment for $15 \mathrm{~h}$ (difference between LV-1C and LV-1/P-2 and between LV-2C and LV-2/P-2, P $\leq 0.01$; difference between LV-1C and LV-1/P-1 and between LV-2C and LV-2/P-1, P $\geq 0.05$ ). LV-nC, LucA-negative control.

as LV transcription, whereas treatment for 30 min with Dex only improves LV transcription.

Effect of Bimaxl on Ima alleviated by Dex (Fig. 3). The increased LucA may be partly attributed to the increasing levels of cytoplasmic Ima. Thus, LucA may be decreased by Bmax1 and the decreased LucA may be elevated through increasing the Dex dose. To verify the above, the $293 \mathrm{~T}$ cells were pretreated with Bmax1, followed by the addition of Dex-1 and Dex-2 30 min prior to transfection. The LucA in the Bmax 1 group was significantly lower compared to that in the $\mathrm{LV}$ alone group $(\mathrm{P} \leq 0.01)$, suggesting that Bimax1 was able to block PIC import through inhibiting Im $\alpha$. In comparison with the Bmax1 group, the LucA in the Dex-1 and Dex-2 groups was significantly elevated $(\mathrm{P} \leq 0.01)$, with the LucA present in the Dex-2 group being higher compared to that in the Dex-1 group $(\mathrm{P} \leq 0.01)$. These results suggest that the elevated LucA may be attributed to the Im $\alpha$ increase mediated by Dex, as well as to the transcription enhanced by Dex.

Effect of RU486 on Ima (Fig. 4). To exclude the effect of transcription on LucA, Dex was replaced with RU486. The 293 T cells, which were pretreated with RU486, followed by the addition of Bmax1 30 min prior to transfection, were transfected with LV. Compared to the RU486 alone group, the LucA in the Bmax1 group was significantly decreased $(\mathrm{P} \leq 0.01)$, 
further confirming that the increased LucA was associated with the increasing cytoplasmic Im $\alpha$ levels.

Effect of Dex on LucA in vivo (Fig. 5). The mice were pretreated with Dex either $30 \mathrm{~min}$ or $15 \mathrm{~h}$ prior to $\mathrm{LV}$ transfection. The 30-min pretreatment aimed to increase cytoplasmic Im $\alpha$ levels, whereas the $15-\mathrm{h}$ pretreatment aimed to suppress immune and inflammatory responses. Compared to the LV-1C and LV-2C groups, the LucA in the LV-1/P-1 and LV-2/P-1 groups, respectively, was not increased $(\mathrm{P} \geq 0.05)$, although the LucA in the LV-1/P-2 and LV-2/P-2 groups was significantly elevated $(\mathrm{P} \leq 0.01)$. These findings suggest that the efficiency of LV transfection depends mainly on suppressing immune and inflammatory responses in vivo.

\section{Discussion}

To the best of our knowledge, this study was the first to demonstrate that the effect of Dex on LV infection is associated with Im $\alpha$, suggesting that Im $\alpha$ may be a novel pathway mediating the effects of Dex on HIV-1 infection.

Dex increases cytoplasmic Ima levels. Im $\alpha$ may bind to the NLS-1 of GR in the absence of gluocorticoid (3). Thus, the cytoplasm contains two types of $\operatorname{Im} \alpha$, namely $\operatorname{Im} \alpha$ and Ima-GR. Following Dex pretreatment, Dex is combined with the NLS-2 of GR to form two new complexes, GR-GR-Dex and Im $\alpha$-GR-Dex (15). Therefore, the cytoplasm contains Dex, GR, Im $\alpha$, Im $\alpha$-GR, Im $\alpha$-GR-Dex and GR-GR-Dex, in a dynamic balance. Dex promotes GR import, thus resulting in the nuclear retention of GR (7,16-18). Subsequently, the GR-GR dimer changes into GR in order to bind GRE, triggering LV gene transcription modulation (19). This may be a mechanism through which Dex pretreatment leads to elevated LucA. Furthermore, the nuclear retention of GR-GR results in decreased cytoplasmic GR levels. According to the balance theory, cytoplasmic GR attenuation inhibits the binding of GR to Im $\alpha$, resulting in increased cytoplasmic Im $\alpha$ levels and improving PIC import. This may be another mechanism underlying the association of elevated LucA with Dex pretreatment. In addition, Dex induces the downregulation of GR (20), which may also help increase cytoplasmic Im $\alpha$ levels.

Bimax1, a peptide with a NLS-like sequence exhibiting a high affinity for Im $\alpha$ (11), may block the import of the nucleus accumbens-associated protein 1 by the Im $\alpha$ pathway (10). In our study, Bimax1 impaired the PIC import that was induced by Dex, resulting in decreased LucA. However, increasing the Dex dose elevated LucA, which was attributed to promoting PIC import and LV gene transcription. RU486 binds to the co-activator pocket of GR (GR1), with domain swapping of the GR3 between the subunits of the GR dimer (21), inducing GR shuttling to the nucleus, without stimulating subsequent events (22). In the present study, Dex was replaced by RU486. LucA was elevated by RU486, but decreased by Bimax1, further suggesting that the change in cytoplasmic Im $\alpha$ levels affects LV infection.

Increased cytoplasmic Im $\alpha$ levels may also result from a change in the GR affinity for Im $\alpha$. The conformational change of the GR D-loop and second helix was shown to confer a change in the GR affinity for Im $\alpha(23,24)$, which was, however, not investigated in the present study.
Increasing cytoplasmic Ima levels favor PIC shuttling to the nucleus. The interaction of Im $\alpha$ with IN and MA results in PIC import $(2,25-27)$, which is followed by the integration of double-strand LV DNA into the target cell genome. Subsequently, the GR accumulated in the nucleus is combined with LV GRE to improve Luci transcription, resulting in increased LucA. Several previous experiments also confirmed that Dex improves LV gene transcription (28-30).

Of note, the inactivation of NLS within IN and MA was previously reported to inhibit viral infection $(2,26)$. Considering that PIC import depends on Ima (31-33), the inactivation of NLS within Im $\alpha$ may also inhibit HIV-1 infection. The majority of the currently available drugs for the treatment of HIV-1 have focused on inhibiting viral entry, viral genome replication and virus-specific proteolysis. However, numerous viruses are able to exploit cellular kinases, facilitating subcellular targeting during infection to achieve drug resistance (34). Accordingly, blocking PIC import may suppress HIV-1 infection through the Im $\alpha$ pathway, although it remains unclear whether inhibiting Im $\alpha$ leads to HIV-1 drug resistance due to the HIV-1 accumulation in the cytoplasm.

In vivo, increasing LucA depends mainly on immunosuppression and anti-inflammation. Following intraperitoneal injection, LV must go through several processes to reach the target cell. During these processes, LV may be sequestered in bypass organs and destroyed by opsonization, immune and inflammatory responses, resulting in significantly impaired gene delivery. Dex is one of the most frequently used immunosuppressive and anti-inflammatory drugs. The binding of Dex to GR suppresses the transcription of numerous cytokines, adhesion molecules and proinflammatory genes via the $\mathrm{NF}-\kappa \mathrm{B}$ pathway (35). Vpr is a coactivator of GR and its effects, including suppressing IL-12 transcription in human monocytes and inducing the apoptosis of human $\mathrm{CD}^{+} \mathrm{T}$ cells and thymocytes, are enhanced by the binding of Dex to GR (36-38). Megadose Dex may preserve lysosomal membrane integrity by rapid non-genomic effects and long-term receptor-dependent genomic events (14). In our study, it was demonstrated that Dex promotes LV infection via the Im $\alpha$ pathway.

In conclusion, LucA may be increased through improving PIC import and LV transcription. The improved PIC import by Dex is associated with $\operatorname{Im} \alpha$, suggesting that $\operatorname{Im} \alpha$ may be a novel pathway mediating the effects of Dex on HIV-1 infection.

\section{Acknowledgements}

This study was funded by a grant from the Less Developed Region of the National Science Foundation of China (no. 81060273). The authors would like to thank Professor Bijun Peng and Wei Dong for their help.

\section{References}

1. Jayappa KD, Ao Z, Yang M, Wang J and Yao X: Identification of critical motifs within HIV-1 integrase required for importin $\alpha 3$ interaction and viral cDNA nuclear import. J Mol Biol 410: 847-862, 2011.

2. Levin A, Armon-Omer A, Rosenbluh J, Melamed-Book N, Graessmann A, Waigmann E and Loyter A: Inhibition of HIV-1 integrase nuclear import and replication by a peptide bearing integrase putative nuclear localization signal. Retrovirology 6: $112,2009$. 
3. Carrigan A, Walther RF, Salem HA, Wu D, Atlas E, Lefebvre YA and Hache RJ: An active nuclear retention signal in the glucocorticoid receptor functions as a strong inducer of transcriptional activation. J Biol Chem 282: 10963-10971, 2007.

4. Lange A, McLane LM, Mills RE, Devine SE and Corbett AH: Expanding the definition of the classical bipartite nuclear localization signal. Traffic 11: 311-323, 2010.

5. Popov S, Rexach M, Ratner L, Blobel G and Bukrinsky M: Viral protein $\mathrm{R}$ regulates docking of the HIV-1 preintegration complex to the nuclear pore complex. J Biol Chem 273: 13347-13352, 1998

6. De Iaco A and Luban J: Inhibition of HIV-1 infection by TNPO3 depletion is determined by capsid and detectable after vira cDNA enters the nucleus. Retrovirology 8: 98-116, 2011.

7. Ou XM, Chen K and Shih JC: Glucocorticoid and androgen activation of monoamine oxidase $\mathrm{A}$ is regulated differently by R1 and Sp1. J Biol Chem 281: 21512-21525, 2006.

8. Russo FO, Patel PC, Ventura AM and Pereira CA: HIV-1 long terminal repeat modulation by glucocorticoids in monocytic and lymphocytic cell lines. Virus Res 64: 87-94, 1999

9. Mitra D, Sikder SK and Laurence J: Role of glucocorticoid receptor binding sites in the human immunodeficiency virus type 1 long terminal repeat in steroid-mediated suppression of HIV gene expression. Virology 214: 512-521, 1995.

10. Okazaki K, Nakayama N, Nariai Y, Nakayama K, Miyazaki K, Maruyama R, Kato H, Kosugi S, Urano T and Sakashita G: Nuclear localization signal in a cancer-related transcriptional regulator protein NAC1. Carcinogenesis 33: 1854-1862, 2012

11. Marfori M, Lonhienne TG, Forwood JK and Kobe B: Structural basis of high-affinity nuclear localization signal interactions with importin- $\alpha$. Traffic 13: 532-548, 2012.

12. Tanaka M, Nishi M, Morimoto M, Sugimoto T and Kawata M: Yellow fluorescent protein-tagged and cyan fluorescent protein-tagged imaging analysis of glucocorticoid receptor and importins in single living cells. Endocrinology 144: 4070-4079, 2003.

13. Xu X, Zhou X, Zhou XW, Zhang Z, Liao MJ, Gao Q and Luo HM Schizandrin prevents dexamethasone-induced cognitive deficits. Neurosci Bull 28: 532-540, 2012.

14. Hinz B and Hirschelmann R: Dexamethasone megadoses stabilize rat liver lysosomal membranes by non-genomic and genomic effects. Pharm Res 17: 1489-1493, 2000.

15. Bledsoe RK, Montana VG, Stanley TB, Delves CJ, Apolito CJ, McKee DD, Consler TG, Parks DJ, Stewart EL, Willson TM, Lambert MH, Moore JT, Pearce KH and Xu HE: Crystal structure of the glucocorticoid receptor ligand binding domain reveals a novel mode of receptor dimerization and coactivator recognition. Cell 110: 93-105, 2002.

16. Johnston PA, Shinde SN, Hua Y, Shun TY, Lazo JS and Day BW: Development and validation of a high-content screening assay to identify inhibitors of cytoplasmic dynein-mediated transport of glucocorticoid receptor to the nucleus. Assay Drug Dev Technol 10: 432-456, 2012.

17. Chen Y, Watson AM, Williamson CD, Rahimi M, Liang C, Colberg-Poley AM and Rose MC: Glucocorticoid receptor and histone deacetylase- 2 mediate dexamethasone-induced repression of MUC5AC gene expression. Am J Respir Cell Mol Biol 47: 637-644, 2012.

18. Charmandari E, Kino T, Ichijo T, Jubiz W, Mejia L, Zachman K and Chrousos GP: A novel point mutation in helix 11 of the ligand-binding domain of the human glucocorticoid receptor gene causing generalized glucocorticoid resistance. J Clin Endocrinol Metab 92: 3986-3990, 2007.

19. Mikuni S, Tamura M and Kinjo M: Analysis of intranuclear binding process of glucocorticoid receptor using fluorescence correlation spectroscopy. FEBS Lett 581: 389-393, 2007.

20. Carey KT, Tan KH, Ng J, Liddicoat DR, Godfrey DI and Cole TJ: Nfil3 is a glucocorticoid-regulated gene required for glucocorticoid-induced apoptosis in male murine T cells Endocrinology 154: 1540-1552, 2013

21. Kauppi B, Jakob C, Farnegardh M, Yang J, Ahola H, Alarcon M, Calles K, Engstrom O, Harlan J, Muchmore S, Ramqvist AK, Thorell S, Ohman L, Greer J, Gustafsson JA, Carlstedt-Duke J and Carlquist M: The three-dimensional structures of antagonistic and agonistic forms of the glucocorticoid receptor ligand-binding domain: RU-486 induces a transconformation that leads to active antagonism. J Biol Chem 278: 22748-27754, 2003.
22. Nelson G, Wilde GJ, Spiller DG, Kennedy SM, Ray DW, Sullivan E, Unitt JF and White MR: NF-kappaB signalling is inhibited by glucocorticoid receptor and STAT6 via distinct mechanisms. J Cell Sci 116: 2495-2503, 2003

23. Stockner T, Sterk H, Kaptein R and Bonvin AM: Molecular dynamics studies of a molecular switch in the glucocorticoid receptor. J Mol Biol 328: 325-334, 2003.

24. Necela BM and Cidlowski JA: A single amino acid change in the first zinc finger of the DNA binding domain of the glucocorticoid receptor regulates differential promoter selectivity. J Biol Chem 279: 39279-39288, 2004.

25. Armon-Omer A, Graessmann A and Loyter A: A synthetic peptide bearing the HIV-1 integrase 161-173 amino acid residues mediates active nuclear import and binding to importin alpha: characterization of a functional nuclear localization signal. J Mol Biol 336: 1117-1128, 2004

26. Haffar OK, Popov S, Dubrovsky L, Agostini I, Tang H, Pushkarsky T, Nadler SG and Bukrinsky M: Two nuclear localization signals in the HIV-1 matrix protein regulate nuclear import of the HIV-1 pre-integration complex. J Mol Biol 299: $359-368,2000$

27. Hearps AC and Jans DA: HIV-1 integrase is capable of targeting DNA to the nucleus via an importin $\alpha / \beta$-dependent mechanism. Biochem J 398: 475-484, 2006.

28. Agudo J, Ruzo A, Kitur K, Sachidanandam R, Blander JM and Brown BD: A TLR and non-TLR mediated innate response to lentiviruses restricts hepatocyte entry and can be ameliorated by pharmacological blockade. Mol Ther 20: 2257-2267, 2012.

29. Karner E, Unger C, Cerny R, Ahrlund-Richter L, Ganss B, Dilber MS and Wendel M: Differentiation of human embryonic stem cells into osteogenic or hematopoietic lineages: a dose-dependent effect of osterix over-expression. J Cell Physiol 218: 323-333, 2009.

30. Gerolami R, Uch R, Jordier F, Chapel S, Bagnis C, Brechot C and Mannoni P: Gene transfer to hepatocellular carcinoma: transduction efficacy and transgene expression kinetics by using retroviral and lentiviral vectors. Cancer Gene Ther 7: 1286-1292, 2000.

31. Resa-Infante P, Jorba N, Zamarreno N, Fernandez Y, Juarez S and Ortin J: The host-dependent interaction of $\alpha$-importins with influenza PB2 polymerase subunit is required for virus RNA replication. PLoS One 3: e3904, 2008.

32. Reid SP, Leung LW, Hartman AL, Martinez O, Shaw ML, Carbonnelle C, Volchkov VE, Nichol ST and Basler CF: Ebola virus VP2 4 binds karyopherin $\alpha 1$ and blocks STAT1 nuclear accumulation. J Virol 80: 5156-5167, 2006.

33. McBride KM, Banninger G, McDonald C and Reich NC: Regulated nuclear import of the STAT1 transcription factor by direct binding of importin-alpha. Embo J 21: 1754-1763, 2002.

34. Alvisi G, Rawlinson SM, Ghildyal R, Ripalti A and Jans DA Regulated nucleocytoplasmic trafficking of viral gene products: a therapeutic target? Biochim Biophys Acta 1784: 213-227, 2008

35. Yamazaki T, Tukiyama T and Tokiwa T: Effect of dexamethasone on binding activity of transcription factors nuclear factor- $\kappa \mathrm{B}$ and activator protein-1 in SW982 human synovial sarcoma cells. In Vitro Cell Dev Biol Anim 41: 80-82, 2005.

36. Mirani M, Elenkov I, Volpi S, Hiroi N, Chrousos GP and Kino T: HIV-1 protein Vpr suppresses IL-12 production from human monocytes by enhancing glucocorticoid action: potential implications of Vpr coactivator activity for the innate and cellular immunity deficits observed in HIV-1 infection. J Immunol 169: 6361-6368, 2002

37. Tomasicchio M, Avenant C, Du Toit A, Ray RM and Hapgood JP: The progestin-only contraceptive medroxyprogesterone acetate, but not norethisterone acetate, enhances HIV-1 Vpr-mediated apoptosis in human $\mathrm{CD}^{+} \mathrm{T}$ cells through the glucocorticoid receptor. PLoS One 8: e62895, 2013.

38. Biswas R, Roy $\mathrm{T}$ and Chattopadhyay U: Prolactin induced reversal of glucocorticoid mediated apoptosis of immature cortical thymocytes is abrogated by induction of tumor. J Neuroimmunol 171: 120-134, 2006. 